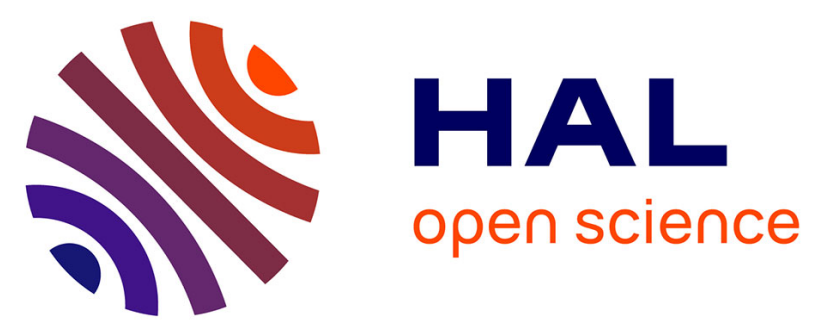

\title{
Empirical Analysis of the Minkowski Distance Order in Geographical Routing Protocols for VANETs
}

Luis Urquiza-Aguiar, Carolina Tripp-Barba, José Estrada-Jiménez, Mónica Aguilar Igartua

\section{- To cite this version:}

Luis Urquiza-Aguiar, Carolina Tripp-Barba, José Estrada-Jiménez, Mónica Aguilar Igartua. Empirical Analysis of the Minkowski Distance Order in Geographical Routing Protocols for VANETs. 13th International Conference on Wired/Wireless Internet Communication (WWIC), May 2015, Malaga, Spain. pp.327-340, 10.1007/978-3-319-22572-2_24. hal-01728809

\section{HAL Id: hal-01728809 https://hal.inria.fr/hal-01728809}

Submitted on 12 Mar 2018

HAL is a multi-disciplinary open access archive for the deposit and dissemination of scientific research documents, whether they are published or not. The documents may come from teaching and research institutions in France or abroad, or from public or private research centers.
L'archive ouverte pluridisciplinaire HAL, est destinée au dépôt et à la diffusion de documents scientifiques de niveau recherche, publiés ou non, émanant des établissements d'enseignement et de recherche français ou étrangers, des laboratoires publics ou privés.

\section{(c)(1)}

Distributed under a Creative Commons Attribution| 4.0 International License 


\title{
Empirical Analysis of the Minkowski Distance Order in Geographical Routing Protocols for VANETs
}

\author{
Luis Urquiza-Aguiar ${ }^{1}$, Carolina Tripp-Barba ${ }^{2}$, José Estrada-Jiménez ${ }^{3}$, and \\ Mónica Aguilar Igartua ${ }^{1}$ \\ 1 Department of Network Engineering, Universitat Politècnica de Catalunya (UPC), \\ C. Jordi Girona 1-3, Barcelona 08034, Spain \\ 2 Faculty of Informatics, Universidad Autonóma de Sinaloa (UAS), De los Deportes \\ Avenue and Leonismo Internacional, Mazatlán 82107, Mexico \\ 3 Department of Electronics, Telecommunications and Networks, Escuela Politécnica \\ Nacional (EPN), Ladrón de Guevara E11 253, Quito 170413, Ecuador \\ \{luis.urquiza, monica.aguilar\}@entel.upc.edu \\ ctripp@uas.edu.mx \\ jose.estrada@epn.edu
}

\begin{abstract}
This paper offers an empirical study of the impact of the way how the distance between two nodes is measured by a geographical routing protocol for VANETs in order to take its forwarding decision. The distance equations used in this work are obtained by setting the order parameter of the Minkowski distance function. Simulation results from the topology of a real city indicate that the use of dominant distance can improve some classical performance metrics like the packet delivery ratio, average number of hops or end-to-end packet delay.
\end{abstract}

Keywords: VANET, geographical routing protocol, Minkowski distance, GPSR.

\section{Introduction}

Vehicular ad hoc networks (VANETs) [5] are seen as a special case of mobile ad hoc networks (MANETs), where nodes are vehicles. Nevertheless, VANETs face particular challenges compared to MANETs, such as faster topology changes, a lower link lifetime or a potentially greater number of nodes taking part in the network, among others. Particularly, the two formers have encouraged researches to propose new routing protocol for VANETs that do not need to construct endto-end paths and that make their routing decision based only on local information.

Geographical routing protocols have emerged as an alternative to the classical topological routing approach. This kind of routing protocols considers the geographical (euclidean measured) distance between nodes in their forwarding criteria. On the other hand, the Minkowski distance function [1] provides a general equation to measure the level of dissimilarity between two points. The Euclidean distance is a particular case of the Minkowski distance function. In this 
work, we analyze the performance impact of a distance-based VANET routing protocol, when it employs distance equations (obtained from the Minkowski distance function) different from the Euclidean one in the computation of its routing metric, when selecting the next hop.

The rest of the paper is organized as follows: section 2 summarizes geographical routing strategy in VANETs and the Minkowski distance family. Then, section 3 describes how using a different distance function changes the forwarding decision made by a distance-based VANET routing protocol. Next, section 4 is devoted to describe the simulation scenario, the evaluation of the different Minkowski distances in the selected protocol and the results obtained from the statistical tests. Finally, conclusions are drawn in Section 5.

\section{Background}

\subsection{Geographical Routing protocols in VANETs}

A widely accepted classification of VANET routing protocols is presented in [9] where routing protocols are divided into two big categories: 1) Topology-based routing protocols perform packet forwarding by using the information of the existing links in the network, and Geographic (position-based), in which nodes make their forwarding decisions based on the position of a packet destination and the positions of the one-hop neighbors. The latter approach was developed taking into account the inherent fast topology changes in VANETs. Many geographical protocols store packets while they do not have a suitable forwarding node, a process called carry-and-forwarding. This specific type of protocols are appropriate for delay-tolerant networks (DTN) and applications due to the delay introduced by the carrying process.

In the following, we summarize the main features of one of the most extended proposals of geographical routing called Greedy Perimeter Stateless Routing (GPSR) [6]. We also describe our proposal, Greedy Buffer Stateless Routing protocol (GBSR) [14], based on GPSR [6], which improves the performance in terms of packet losses.

Greedy Perimeter Stateless Routing (GPSR) [6] operates in two modes: greedy forwarding, which is used by default; and perimeter forwarding, which is used in those regions where greedy forwarding cannot be used. With greedy forwarding, the neighbor geographically closest to the packet's destination (greedy choice) is chosen as the packet's next hop, Figure 1a. When there is not a closest neighbor to destination, GPSR uses the right-hand rule to route packets around voids. The sequence of edges traversed by the right-hand rule is called perimeter, Figure 1b. GPSR poses two important drawbacks: the first one reported in [10] is the use of outdated information; the second one is the inefficient perimeter forwarding scheme [4]. Greedy Buffer Stateless Routing protocol (GBSR) [14] is an improved version of GPSR since GBSR uses a precise knowledge of the neighbors and destination positions. Furthermore, instead of using the perimeter mode, we propose to use a buffer solution. GBSR includes the location information of the neighbors to choose the optimal next forwarding node. The general idea of GBSR 


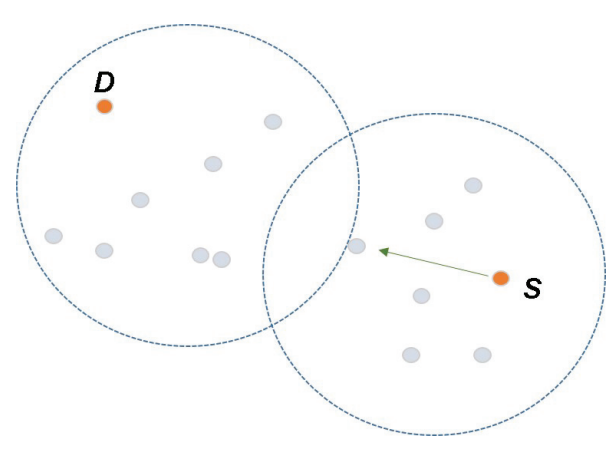

(a) Greedy forwarding mode in GPSR.

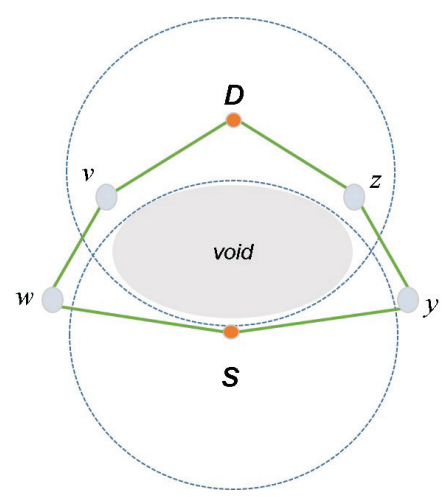

(b) Perimeter forwarding mode in GPSR.

Fig. 1: Forwarding operation modes in Greedy Perimeter Stateless Routing (GPSR).

is to store packets in a local buffer of the node and try to send them later if the node detects a neighbor as a possible forwarding node. A possible forwarding node is any node that satisfies two conditions:

- It is in the transmission range of the current forwarding node. This estimation based on the position an speed of the neighbors.

- It is the nearest neighbor to destination, deciding among all the neighbor nodes.

GBSR tackles the important issue of which neighbors are actually reachable. This feature is of paramount importance to determinate if a neighbor in the neighbor list could be a good forwarding node. Accurate information about the current position of a neighbor has a strong impact in the performance of GBSR, because knowing this information makes the node aware of which neighbors are reliable to be next forwarding nodes. At the same time, it avoids sending packets to an unreachable node.

In order to take a forwarding decision, GBSR uses the same criterion as the original GPSR. It consists of choosing the nearest neighbor to destination based on the geographical distance. However, GBSR stores the packets in a buffer when there is no neighbor that satisfies all the requirements needed to be a next forwarding node. If at least one of the two conditions (i.e., being actually a neighbor and being closer to destination than the current carrier node) required to be the next hop is not satisfied, then packets are stored in the buffer. If the buffer is full, packets are dropped.

GBSR stores packets in the buffer, while it periodically tries to forward them to a new neighbor that meets the requirements to be a proper forwarding node. This period of time was established in $1 \mathrm{sec}$, because this value was frequently enough to detect a topology change. For each destination the packets are stored in arriving order and sent in the same order, so the implemented buffer is a FIFO queue. 


\subsection{Minkowski distance}

A distance function $\delta$ for two n-dimensional points $x$ and $y$ measures how far they are from each other. This is the level of dissimilarity between these two points. A distance measure $\delta(x, y)$ satisfies:

$$
\begin{aligned}
& \delta(x, y)=\delta(y, x) \\
& \delta(x, y) \geq 0 \\
& \delta(x, x)=0
\end{aligned}
$$

The Minkowski distance [1] of order $r$ between the points $x$ and $y$ is defined as:

$$
\delta_{r}(x, y)=\left(\sum_{i=1}^{n}\left|x_{i}-y_{i}\right|^{r}\right)^{1 / r}
$$

If $r<0$, the Minkowski distance function $\delta(2)$ can be seen as a similarity measure instead of quantify how different are two points. Particular cases of the Minkowski distance family are the Manhattan and Euclidean distances. These distances are obtained with the order $r$ equal to 1 and 2, respectively, in the Minkowski distance function $\delta$. When the order $r \rightarrow \infty$ the Minkowski distance function is:

$$
\delta_{r}(x, y)=\lim _{p \rightarrow+\infty}\left(\sum_{i=1}^{n}\left|x_{i}-y_{i}\right|^{p}\right)^{\frac{1}{p}}=\max _{i=1}^{n}\left|x_{i}-y_{i}\right|
$$

This distance is called "dominant" because its value is equal to the maximum of the absolute value of the differences between their components $x$ and $y$.

Figure 2 shows all the points that are at a distance of 1 from the center, which is the definition of a circle in Euclidean distance $(r=2)$. Notice how that circle grows progressively until reaching the square form in the infinity $(r \rightarrow+\infty)$. This is because when $r$ increases, the influence of the highest component $\left|x_{i}-y_{i}\right|^{r}$ in Eq. 2 increases notably compared to the other components in the distance computation. On the other hand, when $r<2$, the area defined by the perimeter is smaller than the Euclidean case $(r=2)$.

\section{Minkowski distance in geographical distance routing metric}

As was explained in Sect. 2, geographical routing protocols mostly base their forwarding decision on the geographical distance from their neighbors to destination. On the other hand, the Minkowski distance function provides a whole family of distances to measure the dissimilarity between two points. This section provides a short explanation of how the forwarding decision is affected by considering alternative ways to measure distance.

The use of order $r \neq 2$ (Euclidean distance) in the Minkowski distance function will affect the operation of a geographical routing protocol in the following parameters: 

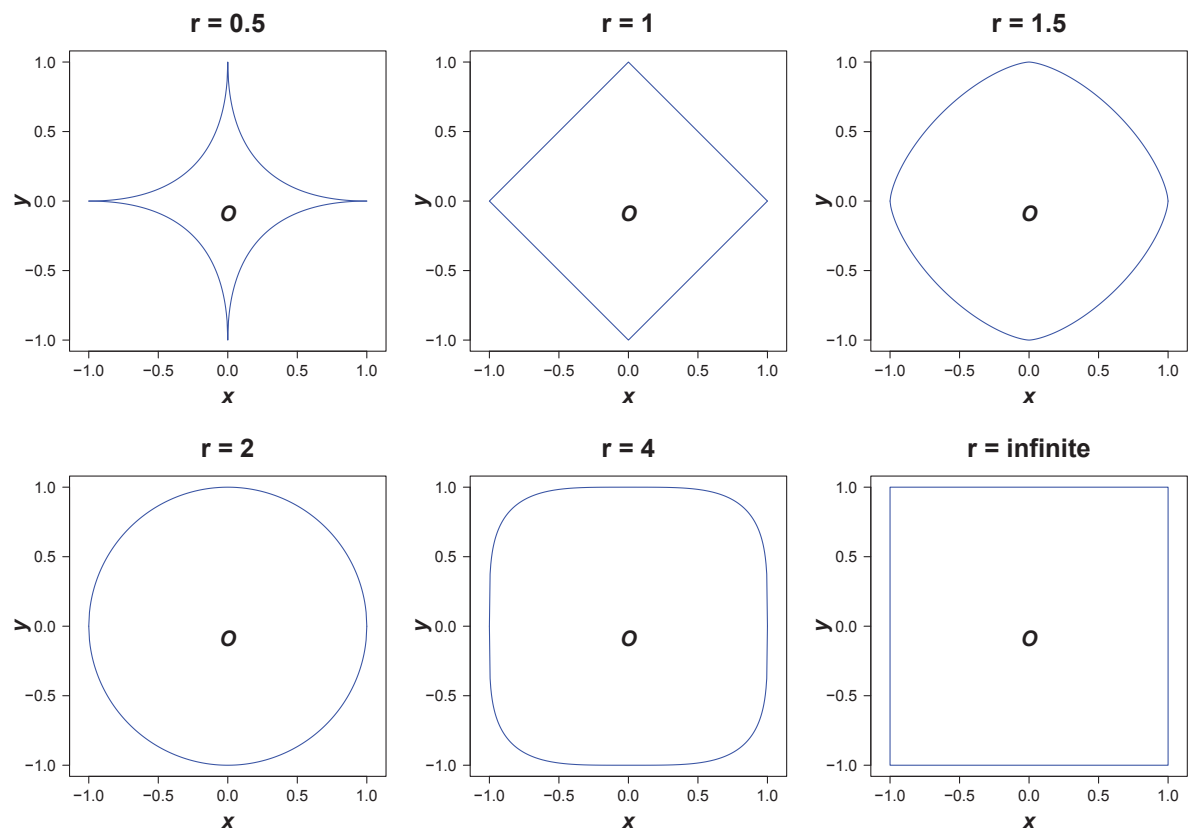

Fig. 2: Points in a 2-dimensional space at a distance of 1 from the center $(O)$ using the Minkowski distance function with different values of the order $r$. Special cases are: Manhattan distance $(r=1)$, Euclidean distance $(r=2)$ and Dominant distance $(r \rightarrow+\infty)$.

1. The size and form of the searching area to find a the next forwarding node.

2. The decision of which neighbor is the closest to destination.

To give an example, Figs. 3 and 4 show a comparison among the use of Euclidean distance $(r=2)$, Manhattan $(r=1)$ and dominant distance $(r \rightarrow+\infty)$, respectively.

As it can be seen in both figures, the Euclidean distance $(r=2)$ between source and destination is the radius of the circle that contains all the nodes closer than the source to the destination, which are called next-hop candidates. For the case of the Manhattan distance $(r=1)$, this area is a diamond. For dominant distance $(r \rightarrow+\infty)$ the area has a square shape. The selected next forwarding node will be in the intersection area that contains the next hop candidates that are also within the coverage area of the source (pink circle around source node in Figs. 3 and 4). Notice that this area changes depending on the Minkowski order $r$. For instance, in the aforementioned figures, red areas mean searching zones only valid if we consider Manhattan distance (Fig. 3) or dominant distance (Fig. 4), but not for Euclidean distance. Conversely, the blue areas, are searching regions only valid for the Euclidean distance. 


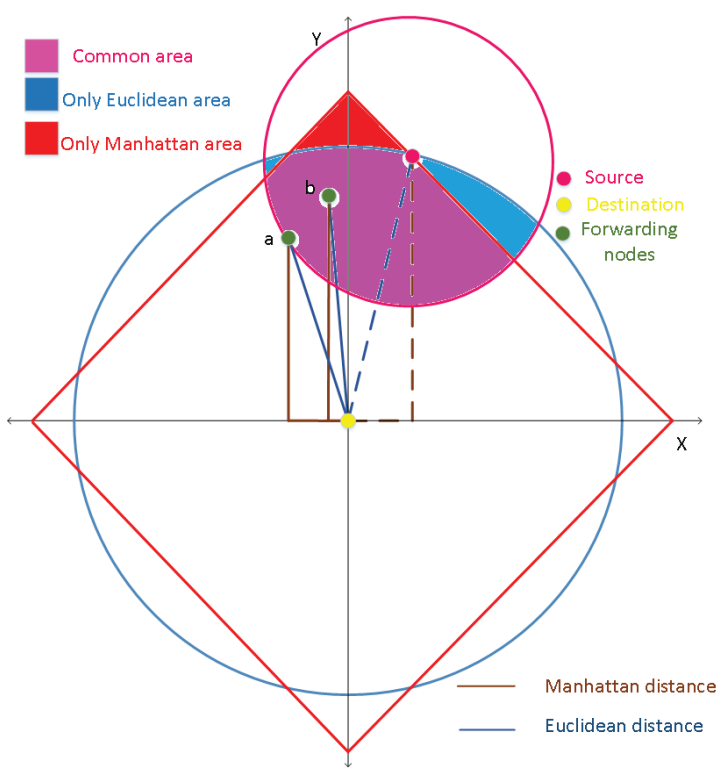

Fig. 3: Euclidean Distance $(r=2)$ vs. Manhattan distance $(r=1)$.

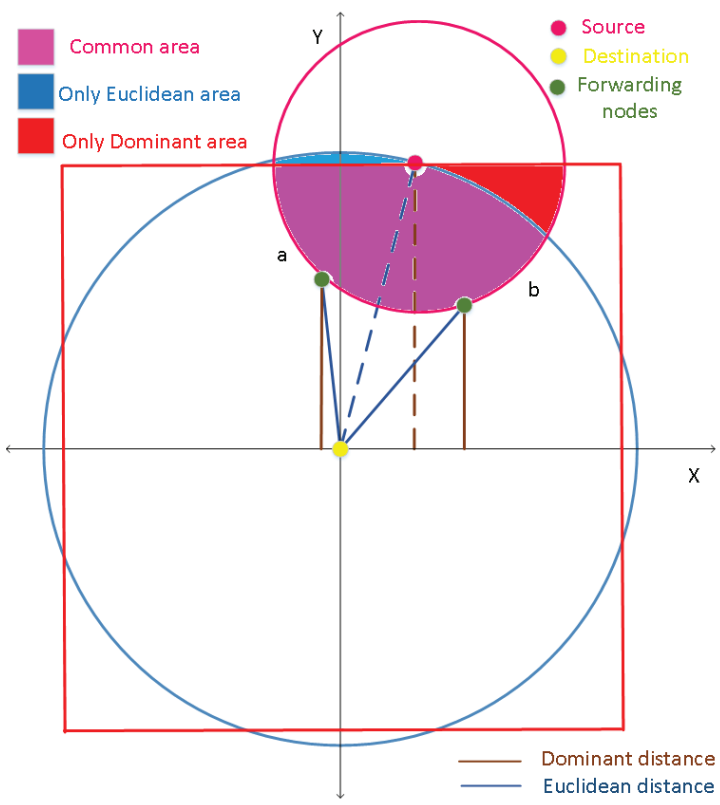

Fig. 4: Euclidean Distance $(r=2)$ vs. dominant distance $(r \rightarrow+\infty)$. 
In both figures, Fig. 3 and Fig. 4 , node $a$ is closer than node $b$ to destination, according to the Euclidean distance. But it is just the opposite if the routing protocol employs the other Minkowski distance orders to make the routing decision. Summarizing, the previous two examples show how the use of other Minkowski distance order values $r$, will affect the influence area where the next hop is selected. Moreover, the selected next forwarding node will not be the same as the chosen one with the Euclidean distance in most of the cases.

\section{Empirical Analysis}

\subsection{Simulation Settings}

The simulation scenario consists of a multi-hop VANET, where we analyzed the impact of the order parameter $r$ of Minkowski distance function in the routing operation of our distance-based proposal GBSR [14]. To do this, we carried out several simulations using the Estinet Network Simulator and Emulator [2]. Estinet is a simulator that includes the standard IEEE 802.11p and a simple and accurate way to design VANET realistic scenarios.

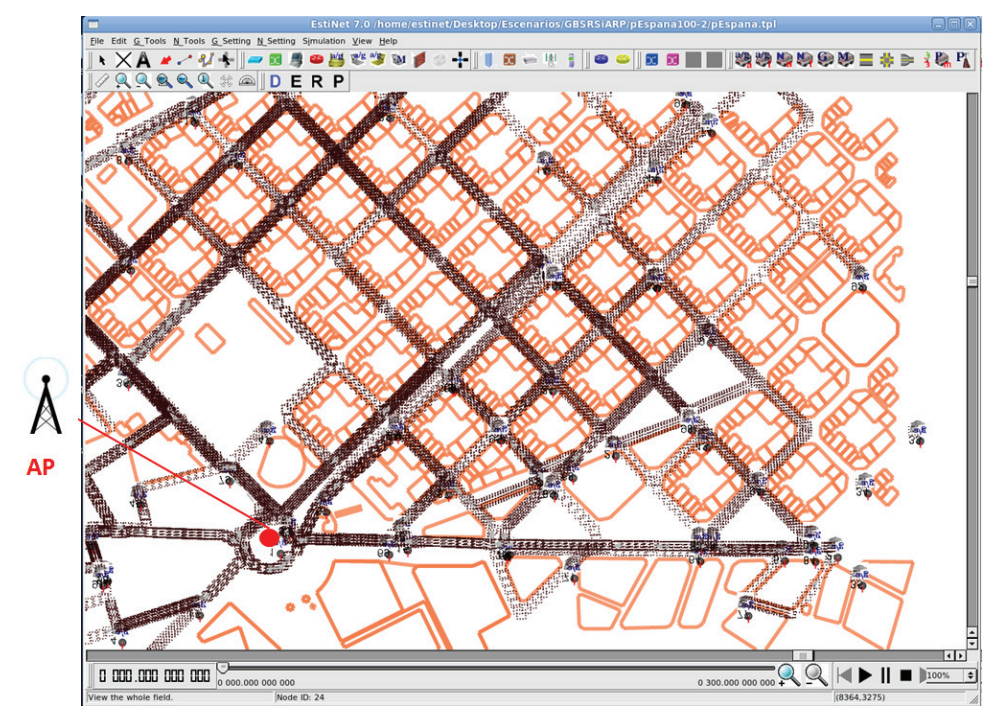

Fig. 5: Simulation scenario. Eixample district of Barcelona, Spain, with an Access Point $(A P)$. Buildings (orange lines) from OpenStreetMap are included.

We used a real scenario of $1.5 \mathrm{~km}^{2}$ taken from the Eixample district of Barcelona (see Fig. 5) to model an urban Manhattan-style scenario, which consists of streets and crossroads. In our realistic scenario, the mobility model was obtained with CityMob for Roadmaps (C4R) [3], a mobility generator that uses 
the SUMO engine [7]. C4R is able to import maps directly from the OpenStreetMap [11] and to generate NS-2 compatible traces. Furthermore, the scenarios have building information (orange lines in Fig. 5) extracted from the OpenStreetMap using the SUMO tools. We exported the NS-2 traces to be compatible with Estinet and the buildings information using our own translating software, available at www.lfurquiza.com/research/estinet [15].

We considered two amounts of vehicles: 100 and 150 vehicles, which correspond to densities of 67 and 100 vehicles per $\mathrm{km}^{2}$, respectively. There was one fixed destination, the access point (henceforth called AP) in Fig. 5, that receives vehicles' traffic information.

We used a single AP in the scenario because in this way we obtained a long range of route lengths, which depends on the position of the source vehicles in the scenario. All nodes sent 1000-byte packets every $T$ seconds to the unique destination during 300 seconds. $T$ follows a uniform distribution from 2 to 6 seconds. Simulations were carried out using the IEEE $802.11 \mathrm{p}$ standard on physical and MAC layers.

All the figures are presented with confidence intervals (CI) of 95\%, obtained from 20 simulations per each density value and order parameter $r$ using different movement traces per each simulation. Table 1 summarizes the main simulation settings.

\begin{tabular}{cc}
\hline Parameter & Value \\
\hline Number of nodes & 100 and 150 vehicles \\
Map zone \& Area & Eixample district of Barcelona, 1.5 km x 1 km \\
Path loss model & Empirical IEEE $802.11 \mathrm{p}$ radio shadowing [13] \\
Fading model & Rician (LOS) and Rayleigh (not in LOS) \\
Power transmission & $23 \mathrm{dbm}$ \\
Receiving sensing & $-82 \mathrm{dbm}(\sim 400 \mathrm{~m}$ in LOS) \\
Mobility generator & SUMO $[7] / \mathrm{C} 4 \mathrm{R}[3]$ \\
Mobility model & Krauss $[8]$ \\
Max speed & $60 \mathrm{~km} / \mathrm{h}$ \\
MAC specification & IEEE $802.11 \mathrm{p}$ \\
Bandwidth & $6 \mathrm{Mbps}$ \\
Simulation time & $300 \mathrm{sec}$ \\
Inter-packet generation time & $T \sim \mathrm{U}(2,6) \mathrm{s} \mathrm{E}(T)=4 \mathrm{~s}$ \\
Packet size & $1000 \mathrm{bytes}$ \\
Routing protocol & $\mathrm{GBSR}[14]$ \\
Minkowski Order parameter $r$ & $0.5,1,1.5,2,2.5,3,4,+\infty$ \\
GPS precision & $10 \mathrm{~m}$ \\
\hline
\end{tabular}

Table 1: Simulation settings.

\subsection{Simulation Results}

In this section, we present some results from comparing the simulations of GBSR using different values for the order parameter $r$. The evaluation is focused on 
three widely-used metrics applied to the performance analysis of VANET routing protocols. These metrics are the percentage of packet losses, the average packet delay and the average number of hops for the packets to reach destination. Figures 6, 7 and 8 depict these results for the two node densities under consideration.

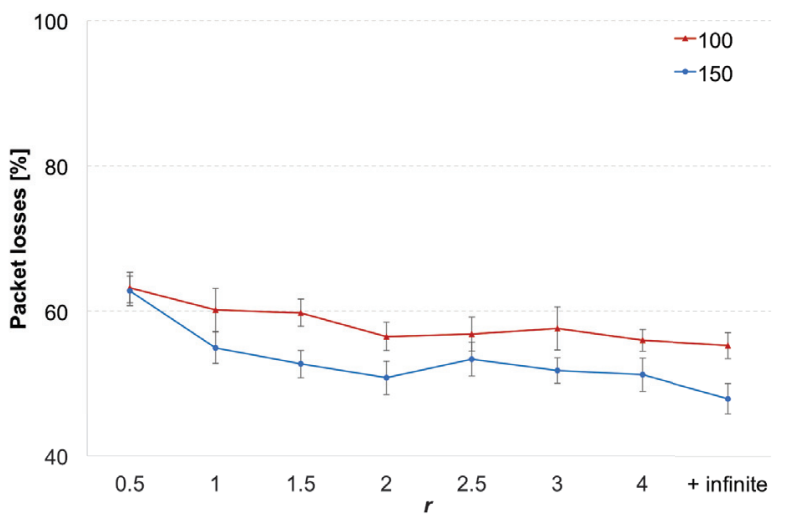

Fig. 6: Percentage of packet losses.

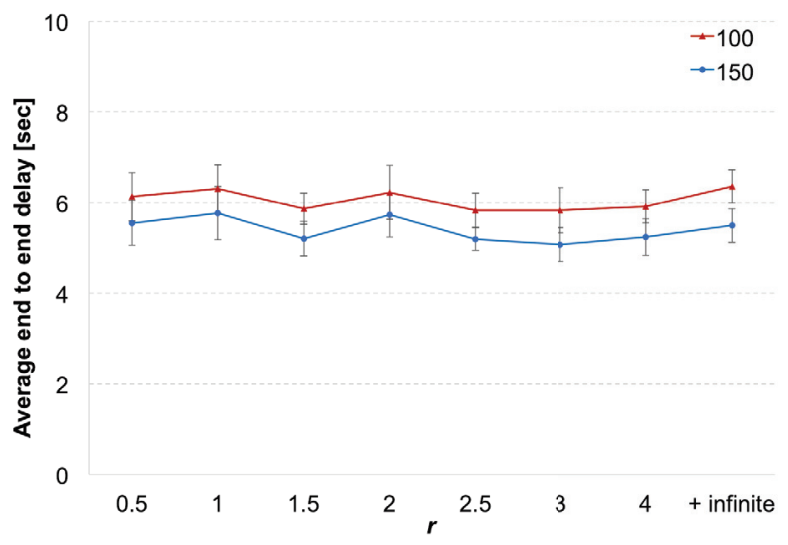

Fig. 7: Average end-to-end packet delay.

As it can be seen from Fig. 6, when the routing protocol uses the Minkowski distance function (see. Eq. 2) with $r<2$, the increase in the percentage of packet losses is considerable compared to the losses when $r=2$ for both vehicle densities. Notice that small $r$ values implies that each $\left|x_{i}-y_{i}\right|^{r}$ component has a similar contribution in the computation of the Minkowski distance $\delta_{r}(x, y)$. Notice also that, for the these values of $r$ (i.e., $r \in\{0.5,1,1.5\}$ ), the average endto-end delay in Fig. 7 are similar among them. However, the averages number of hops are very different (see Fig. 8) in the three cases. The measure of distance with $r=1.5$ produces slightly shorter routes than the ones obtained with Euclidean distance $(r=2)$. Manhattan distance $r=1$ has by far the longest 


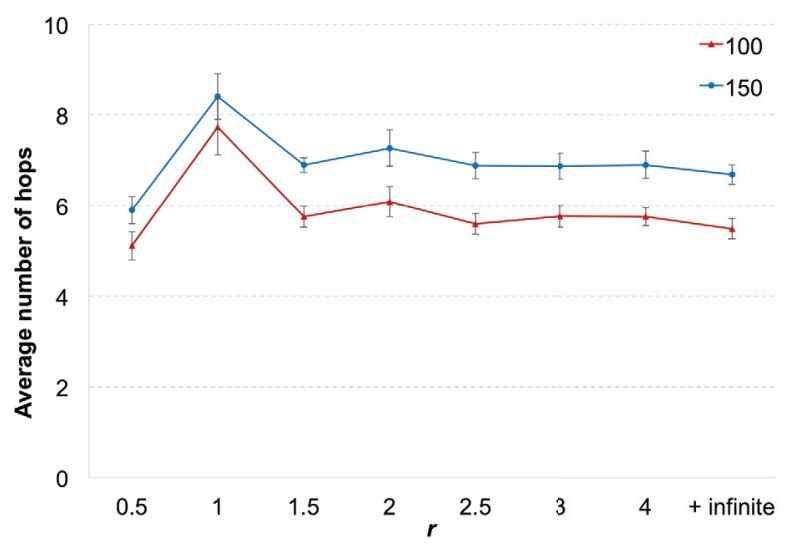

Fig. 8: Average number of hops.

average number of hops in a route. This fact explains the high percentage of packet losses for Manhattan distance, because more hops entails higher chances of packet collision or packet error reception. On the contrary, the Minkowski distance with $r=0.5$ has the shortest average number of hops, at the cost of the highest packet losses. These facts could be explained due to the shape of the searching area for $r=0.5$ (see Fig. 2), which in most of the cases will differ more than any other searching area from the Euclidean circle.

To analyze the performance metrics for $r>2$, we employ statistical tests because the relationship of the results among the distances is not so evident as it is in the previous case. For each density of vehicles we will use pairwise Wilcoxon statistical test [12] to check whether the differences between the results obtained with all the $r \geq 2$ and those coming from the simulation with Euclidean distances are statistically significant. Tables. 2, 3 and 4 summarize the results of this test for the three metrics (i.e., percentage of packet losses, average end-to-end delay, and average number of hops in a path), and grouped by the two vehicle density. The outcome of a statistical test is a probability called the $p$-value (fourth column of tables. 2, 3 and 4), which is compared with a threshold named the significance level. If the $p$-value is lower than the significance level, then the difference between both performance metrics are statistically significant (fifth column in the tables). We employed a significance level on each test of 0.025 , to obtain an overall error probability among the four pairwise comparison per metric of 0.10 (i.e., $0.025 \times 4$ ). The results of the statistical tests indicate that when there is a low vehicle density scenario (100 vehicles), there is no improvement or degradation on the percentage of packet losses and average end-to-end packet delay compared with the results obtained using the Euclidean distance (see tables. 2 and 3). However, there is a statistically significant difference in the average path length when the routing protocol GBSR employs a Minkowski distance with order $r=2.5$ or the dominant distance $(r \rightarrow+\infty)$, as can be seen in the fifth and eighth rows of table 4 . It means that the use of $r \in\{2.5,+\infty\}$ in our routing protocol GBSR for our low-density scenario provides the same quality of service (packet losses and delay) through shorter paths. 


\begin{tabular}{|c|c|c|c|c|c|}
\hline $\begin{array}{c}\text { Vehicle } \\
\text { Density }\end{array}$ & $\begin{array}{c}\text { Pairwise } \\
(r, 2)\end{array}$ & $\begin{array}{l}\text { Standardized } \\
\text { Test Statistic }\end{array}$ & $\begin{array}{c}p \text {-Value } \\
1 \text { Side }\end{array}$ & $\begin{array}{c}\text { Is the Difference } \\
\text { Significant } \\
(p \text {-Value }<0.025) ?\end{array}$ & $\begin{array}{l}\text { Median of } \\
\text { Differences }\end{array}$ \\
\hline \multirow{4}{*}{100} & 2.5 & -0.336 & 0.378 & No & $1.088 \%$ \\
\hline & 3 & -0.896 & 0.194 & No & $2.52 \%$ \\
\hline & 4 & -0.485 & 0.324 & No & $0.158 \%$ \\
\hline & $+\infty$ & -1.381 & 0.088 & No & $-1.441 \%$ \\
\hline \multirow{4}{*}{150} & 2.5 & -2.091 & 0.018 & Yes & $2.549 \%$ \\
\hline & 3 & -0.971 & 0.174 & No & $1.024 \%$ \\
\hline & 4 & -0.299 & 0.392 & No & $1,738 \%$ \\
\hline & $+\infty$ & -2.24 & 0.012 & Yes & $-3.096 \%$ \\
\hline
\end{tabular}

Table 2: $p$-values of Wilcoxon signed rank test for a pairwise comparison of the effect of the Minkowski distance order $r$ for the packet losses metric.

\begin{tabular}{|c|c|c|c|c|c|}
\hline $\begin{array}{l}\text { Vehicle } \\
\text { Density }\end{array}$ & $\begin{array}{c}\text { Pairwise } \\
\quad(r, 2)\end{array}$ & $\begin{array}{l}\text { Standardized } \\
\text { Test Statistic }\end{array}$ & $\begin{array}{l}p \text {-Value } \\
1 \text { Side }\end{array}$ & $\begin{array}{c}\text { Is the Difference } \\
\text { Significant } \\
(p \text {-Value }<0.025) ?\end{array}$ & $\begin{array}{l}\text { Median of } \\
\text { Differences }\end{array}$ \\
\hline \multirow{4}{*}{100} & 2.5 & -1.232 & 0.115 & No & $-0.483 \mathrm{~s}$ \\
\hline & 3 & -1.307 & 0.101 & No & $-0.341 \mathrm{~s}$ \\
\hline & 4 & -1.083 & 0.147 & No & $-0.158 \mathrm{~s}$ \\
\hline & $+\infty$ & -0.859 & 0.205 & No & $0.037 \mathrm{~s}$ \\
\hline \multirow{4}{*}{150} & 2.5 & -2.427 & 0.007 & Yes & $-0.500 \mathrm{~s}$ \\
\hline & 3 & -2.763 & 0.002 & Yes & $-0.584 \mathrm{~s}$ \\
\hline & 4 & -2.203 & 0.013 & Yes & $-0.409 \mathrm{~s}$ \\
\hline & $+\infty$ & -1.269 & 0.108 & No & $-0.186 \mathrm{~s}$ \\
\hline
\end{tabular}

Table 3: $p$-values of Wilcoxon signed rank test for a pairwise comparison of the effect of the Minkowski distance order $r$ for the average end-to-end packet delay metric.

\begin{tabular}{|c|c|c|c|c|c|}
\hline $\begin{array}{l}\text { Vehicle } \\
\text { Density }\end{array}$ & $\begin{array}{c}\text { Pairwise } \\
\quad(r, \mathscr{2})\end{array}$ & $\begin{array}{l}\text { Standardized } \\
\text { Test Statistic }\end{array}$ & $\begin{array}{c}p \text {-Value } \\
1 \text { Side }\end{array}$ & $\begin{array}{c}\text { Is the Difference } \\
\text { Significant } \\
(p \text {-Value }<0.025) ?\end{array}$ & $\begin{array}{l}\text { Median of } \\
\text { Differences }\end{array}$ \\
\hline \multirow{4}{*}{100} & 2.5 & -2.837 & 0.002 & Yes & -0.367 hops \\
\hline & 3 & -1.680 & 0.049 & No & -0.1635 hops \\
\hline & 4 & -1.867 & 0.03 & No & -0.228 hops \\
\hline & $+\infty$ & -3.173 & 0.0005 & Yes & -0.515 hops \\
\hline \multirow{4}{*}{150} & 2.5 & -1.829 & 0.035 & No & -0.337 hops \\
\hline & 3 & -2.165 & 0.015 & Yes & -0.0136 hops \\
\hline & 4 & -1.979 & 0.024 & Yes & -0.66 hops \\
\hline & $+\infty$ & -3.323 & 0.0005 & Yes & -0.11 hops \\
\hline
\end{tabular}

Table 4: $p$-values of Wilcoxon signed rank test for a pairwise comparison of the effect of the Minkowski distance order $r$ for the average number of hops metric.

For the intermediate vehicle density scenario (150 vehicles), our results indicate that only when GBSR used the dominant distance to make the forwarding decision, the percentage of packet losses shows a statistically significant reduction 
(around 3\%, see table 2) compared with GBSR employing classical Euclidean distance. This packet losses reduction is obtained without increasing the average end-to-end delay, which remains statistically equal (eighth row in table 3 ). Moreover, this reduction of packet losses and similar delay, using the dominant distance comes with the use of shorter paths than the created by GBSR using Euclidean distance (around 0.18 hops, see table 4 ). In the cases of $r=3$ and $r=4$, both distances do not have differences with the Euclidean in the percentage of packet losses. Nonetheless, regarding to the average end-to-end and path length, there are statistically significant improvements as shown in rows sixth and seventh in tables 3 and 4. The Minkowski's order $r=2.5$ in the intermediate vehicle density for the percentage of packet losses provides worse marks than the obtained with the classical Euclidean distance and only improve the average end-to-end packet delay.

To conclude with, the simulation results show that it is possible to improve some performance metrics of our geographical routing protocol GBSR, if a different distance equation is used by the routing protocol. Particularly, the dominant distance $(r \rightarrow+\infty)$ equation outperforms the traditional results $(r=2)$ in terms of packet losses and average number of hops for intermediate vehicle density and only the latter in low density areas. However, the result differences in the performance metrics between Euclidean and other distances are not high.

\section{Conclusions and Future work}

In this work we have tested the impact of using different distance equations in the forwarding decision of a geographical routing protocol for VANETs. The distance equations were obtained through the Minkowski distance function, modifying the value of its order parameter. Our results in a realistic urban scenario, indicate that for low and intermediate vehicle densities, the use of the dominant distance $(r \rightarrow+\infty)$ in the routing decision leads to the creation of paths shorter than the ones obtained by the routing protocol employing the traditional Euclidean distance $(r=2)$. Moreover, for the intermediate density (150 vehicles), the routing protocol improved its packet delivery ratio without increasing delay. Also, for intermediate densities, the Minkowski's order 3 and 4 were able to reduce the end-to-end packet delay keeping the same packet losses level. Nevertheless, our results show that the performance differences between euclidean distance are not far from the best ones obtained by other Minkowski $r$ value.

Future work include performing this same comparison in other city topologies in order to find out whether the performance metric differences among the Minkowski's order depends on the layout of the city. Additionally, we are interested in developing a geographical routing protocol that combines some Minkowski distances like Manhattan, Euclidean and dominant to take the forwarding decision.

Acknowledgments. This work was partly supported by the Spanish Government through projects TEC2010-20572-C02-02 Continuity of Service, Security and QoS for Transportation Systems "CONSEQUENCE" and TEC 2013- 
47665-C4-1-R Emergency Response In Smart Communities "EMRISCO". Luis Urquiza-Aguiar is the recipient of a full scholarship from the Secretaria Nacional de Educación Superior, Ciencia y Tecnología (SENESCYT) with the sponsorship of Escuela Politécnica Nacional (EPN) (Ecuador). Carolina Tripp-Barba would like to thank the support of the "Universidad Autónoma de Sinaloa (Mexico)".

\section{References}

1. Borg, I., Groenen, P.: Modern Multidimensional Scaling - Theory and Applications. Springer New York, New York, second edn. (2005)

2. Estinet-Technologies: EstiNet 7 Network Simulator and Emulator (2015), http: //www. estinet. com/products.php?lv1=13\&sn=15

3. Fogue, M., Garrido, P., Martinez, F.J., Cano, J.C., Calafate, C.T., Manzoni, P.: A realistic simulation framework for vehicular networks. In: 5th International ICST Conference on Simulation Tools and Techniques. pp. 37-46. ACM, Brussels, Belgium (2012), http://dl .acm.org/citation. cfm?id=2263019.2263025

4. Gerla, M., Kleinrock, L.: Vehicular networks and the future of the mobile internet. Computer Networks 55(2), 457-469 (2011), http://dx.doi.org/10.1016/j. comnet.2010.10.015

5. Hartenstein, H., Laberteaux, K., Ebrary, I.: VANET: vehicular applications and inter-networking technologies. Wiley Online Library (2010)

6. Karp, B., Kung, H.T.: GPSR Greedy perimeter stateless routing for wireless networks. In: 6th annual international conference on Mobile computing and networking. pp. 243-254. ACM Press, New York, USA (2000), http://dl.acm.org/ citation. $\mathrm{cfm}$ ? id=345910.345953

7. Krajzewicz, D., Erdmann, J., Behrisch, M., Bieker, L.: Recent development and applications of SUMO - Simulation of Urban MObility. International Journal On Advances in Systems and Measurements 5(3\&4), 128-138 (2012)

8. Krauss, S., Wagner, P., Gawron, C.: Metastable states in a microscopic model of traffic flow. Phys. Rev. E 55(5), 5597-5602 (1997), http://link.aps.org/doi/10. 1103/PhysRevE.55.5597

9. Lee, K., Lee, U., Gerla, M.: Survey of Routing Protocols in Vehicular Ad Hoc Networks. In: Advances in Vehicular Ad-Hoc Networks: Developments and Challenges, pp. 149-170. Information Science Reference (2009)

10. Naumov, V., Baumann, R., Gross, T.: An evaluation of inter-vehicle ad hoc networks based on realistic vehicular traces. In: 7th ACM international symposium on Mobile ad hoc networking and computing. pp. 108-119. ACM Press, New York, USA (2006), http://dl.acm.org/citation.cfm?id=1132905.1132918

11. OpenStreetMap: (2015), http://www.openstreetmap.org/

12. Sheskin, D.: Handbook of Parametric and Nonparametric Statistical Procedures. CHAPMAN \& HALL/CRC, Boca Raton, USA, second edn. (2000)

13. Sommer, C., Eckhoff, D., German, R., Dressler, F.: A computationally inexpensive empirical model of ieee 802.11 p radio shadowing in urban environments. In: Eighth International Conference on, Wireless On-Demand Network Systems and Services. pp. 84-90. IEEE Press (2011)

14. Tripp Barba, C., Urquiza Aguiar, L., Aguilar Igartua, M.: Design and evaluation of GBSR-B, an improvement of GPSR for VANETs. IEEE Latin America Transactions 11(4), 1083 - 1089 (2013)

15. Urquiza, L.: Developed code for Estinet simulator (2015), http://www . Ifurquiza. $\mathrm{com} / \mathrm{research} /$ estinet 